\title{
Assessment of Atrial Conduction Times in Patients with Newly Diagnosed Parkinson's Disease
}

\author{
Yiğit Çanga $\mathbb{D}^{1},{ }^{1}$ Ayşe Emre, ${ }^{1}$ Gülbün Asuman Yüksel, ${ }^{2}$ Mehmet Baran Karataş, ${ }^{1}$ \\ Nizamettin Selçuk Yelgeç, ${ }^{1}$ Ufuk Gürkan, ${ }^{1}$ Ali Nazmi Çalık ${ }^{D},{ }^{1}$ Hülya Tireli, ${ }^{2}$ and Sait Terzi ${ }^{1}$ \\ ${ }^{1}$ Department of Cardiology, Dr. Siyami Ersek Cardiovascular and Thoracic Surgery Center, Istanbul, Turkey \\ ${ }^{2}$ Department of Neurology, Haydarpasa Numune Training and Research Hospital, Istanbul, Turkey \\ Correspondence should be addressed to Yiğit Çanga; canga81@hotmail.com
}

Received 3 March 2018; Accepted 12 June 2018; Published 16 July 2018

Academic Editor: Jan Aasly

Copyright (C) 2018 Yiğit Çanga et al. This is an open access article distributed under the Creative Commons Attribution License, which permits unrestricted use, distribution, and reproduction in any medium, provided the original work is properly cited.

Background. An increased risk of ischemic stroke has been reported in patients with Parkinson's disease (PD). Atrial fibrillation (AF) is strongly associated with ischemic stroke. Prolonged atrial electromechanical delay (EMD) is an independent predictor for the development of AF. Aims. The aim of the present study was to evaluate the atrial conduction parameters in patients with PD and to assess their relation with the severity of PD. Study design. We prospectively enrolled 51 consecutive patients with newly diagnosed PD and 31 age- and sex-matched non-PD subjects. Methods. To assess atrial electromechanical coupling (PA), the time intervals from the onset of $\mathrm{p}$ wave on ECG to the late diastolic wave at the septal (PAs) and lateral (PAl) mitral annulus and lateral tricuspid annulus (PAt) were measured on Tissue Doppler Echocardiography (TDE). The difference between PAs-PAl, PAs-PAt, and PAl-PAt were defined as left intra-atrial, right intra-atrial, and interatrial EMD, respectively. P-wave dispersion (PWD) was calculated from the 12-lead ECG. Results. PWD, PAs, PAl, and PAt durations were significantly prolonged in the PD group (all $p<0.001)$. Interatrial, right, and left intra-atrial EMD were also significantly longer in PD patients $(p<0.001, p<0.001$ and $p=0.002$, resp.). There were significant positive correlations between disease severity (UPDRS score) and PWD ( $r=0.34$, $p=0.041)$, left intra-atrial $(r=0.39, p=0.005)$, and interatrial EMD $(r=0.35, p=0.012)$. By multivariate analysis, PWD (OR: 1.13, 95\% CI: $1.02-1.25 ; p=0.017$ ), LA volume index (OR: 1.19, 95\% CI: $1.02-1.37 ; p=0.021$ ), left intra-atrial (OR: $1.12,95 \%$ CI: $1.01-1.24 ; p=0.041$ ), and interatrial EMD (OR: 1.08, 95\% CI: 1.01-1.16; $p=0.026$ ) were found as independent predictors of PD. Conclusion. Atrial conduction times were longer and correlated with the severity of disease in PD patients. Prolonged inter- and intra-atrial-EMD intervals were also found as independent correlates of PD. These findings may suggest an increased predisposition to atrial fibrillation in PD.

\section{Introduction}

Parkinson's disease (PD) has been associated with an increased risk of ischemic stroke and stroke-related mortality [1-3]. A recent population-based, propensity score-matched longitudinal follow-up study demonstrated that newly diagnosed PD was related with an increased risk of developing ischemic stroke [4]. Patients with atrial fibrillation have about 3- to 5 -fold higher risk of stroke even after adjustment for risk factors [5]. Atrial fibrillation has been associated with stroke in different patient populations [6]. Prediction of atrial fibrillation may be crucial for risk stratification of PD patients with regard to ischemic stroke.
Prolonged atrial conduction times have been related to both onset and recurrence of atrial fibrillation $[7,8]$. Tissue Doppler Echocardiography (TDE) has been used to determine atrial electromechanical coupling and electromechanical delay (EMD) intervals between different regions as indicators of electrical and/or structural remodeling of atria and as predictors of atrial fibrillation [9]. Regional changes in atrial conduction times might have a different influence on surface $\mathrm{p}$ waves leading to an interlead variation in $\mathrm{p}$-wave duration called p-wave dispersion (PWD) [10]. In the present study, we investigated atrial conduction parameters in patients with newly diagnosed $\mathrm{PD}$ and also evaluated their relationship with the severity of PD. 


\section{Methods}

2.1. Study Population. Fifty-one consecutive patients with newly diagnosed PD and 31 age- and sex-matched non-PD subjects were prospectively enrolled between January 1st, 2015 and December 31, 2015. Patients with PD were diagnosed according to the UK Parkinson's Disease Society Brain Bank Criteria [11]. The severity of PD was assessed by Unified Parkinson's Disease Rating Scale (UPDRS) [12]. Patients with a previous diagnosis of PD, stroke, other extrapyramidal disease, abnormal movement disorder, cerebral degeneration, cardiac arrhythmia, valvular heart disease, heart failure, coronary artery disease, chronic obstructive pulmonary disease, chronic renal failure, thyroid disease, active infectious disease, poor echocardiographic image quality, and a history of cardiac surgery or implanted device were excluded from the study. Baseline history, medication, and electrocardiography were recorded at the beginning of enrollment. PWD was defined as the difference between the maximum and minimum p-wave duration from multiple surface ECG leads [10]. The study was approved by the local ethics committee. Written informed consents were obtained from all participants.

2.2. Echocardiography. All patients were evaluated by transthoracic $M$ mode, two dimensional, pulsed wave, continuous wave, colour flow, and TDE using the GE Vivid 3 system (GE Vingmed, Horten, Norway) with a $2.5-3.5 \mathrm{MHz}$ transducer. Continuous single lead ECG was obtained from each participant during echocardiography.

LV diameter and wall thickness were measured by M-mode echocardiography. LV ejection fraction was calculated by Simpson's method according to the American Society of Echocardiography guidelines [13]. The mitral valve inflow pattern (E wave, $\mathrm{A}$ wave, E-wave deceleration time, E/A ratio, and isovolumic relaxation time) was measured by pulsed wave Doppler. LA volume was obtained from apical four and two chamber views by a disc method and indexed to body surface area [13].

TDE was performed by adjusting the pulsed Doppler signal filters to acquire the Nyquist limit of $15-20 \mathrm{~cm} / \mathrm{s}$ and using the minimal optimal gain. Motions were recorded simultaneously with electrocardiogram in lead II to assess the relation between atrial electrical phases and myocardial motion. To assess atrial electromechanical coupling (PA), the time intervals from the onset of $\mathrm{p}$ wave on ECG to the late diastolic wave at the septal (PAs), and lateral (PAl) mitral annulus and lateral tricuspid annulus (PAt) were measured on TDE. The difference between PAs-PAl, PAs-PAt, and PAl-PAt were defined as left intra-atrial, right intra-atrial and interatrial EMD, respectively [7, 9]. Echocardiographic measurements were performed by two cardiologists. Patients with $a>5 \%$ difference between the measurements of two cardiologists were not included.

2.3. Statistical Analysis. Statistical analysis was performed using SPSS 16.0 (SPSS, Inc. Chicago, Illinois). A two-tailed $p$ value $<0.05$ was considered statistically significant.
Categorical variables were expressed as frequencies (percentages). Continuous variables were presented as mean \pm standard deviation (tested for normality with Shapiro-Wilk test). Categorical variables were compared using the chisquare or Fischer's exact tests. Group means for continuous variables were compared using independent sample $t$-test. Pearson's correlation test was performed to assess the correlation between atrial conduction times and the severity of PD. Multivariate regression analysis was used to identify independent predictors of PD. Age, gender, hypertension, diabetes, LA volume index, PWD, and EMD intervals were included in the multivariate models.

\section{Results}

Fifty-one consecutive PD patients (mean age: $66.3 \pm 12.4$ years and $71 \%$ men) and 31 non-PD subjects (mean age: $69.8 \pm 12.7$ years and $52 \%$ men) entered the study. Mean UPDRS score of the PD group was $35.3 \pm 17.9$ (range, 10 to 75). Baseline demographic and clinical characteristics of the $\mathrm{PD}$ and non-PD groups are provided in Table 1. There was no statistically significant difference in all the baseline characteristics between the PD and non-PD groups.

Echocardiographic parameters are provided in Table 2. LA volume index was significantly higher in the PD group $(p=0.006)$. Mitral E/A was lower and mitral E-wave deceleration time was higher in the PD group, but both failed to reach statistical significance $(p=0.057$ and $p=0.058$, resp.). The remaining standard echocardiographic parameters were comparable between the two groups.

Atrial conduction time intervals are shown in Table 2. PWD, PAs, PAl, and PAt durations were significantly prolonged in the PD group (all $p<0.001$ ). Left intra-atrial, right intra-atrial, and interatrial EMD were significantly longer in PD patients $(p<0.001, p<0.001$, and $p=0.002$, resp.). PWD showed significant correlations with left intraatrial $(r=0.57, p<0.001)$ and interatrial EMD $(r=0.54$, $p<0.001)$.

There were significant positive correlations between disease severity (UPDRS score) and PWD $(r=0.36, p=0.008)$, left intra-atrial $(r=0.34, p=0.015)$, and interatrial EMD $(r=0.43, p=0.002)$ (Figure 1$)$. By multivariate analysis, PWD (OR: 1.13, 95\% CI: 1.02-1.25; $p=0.017$ ), LA volume index (OR: 1.19, 95\% CI: 1.02-1.37; $p=0.021$ ), left intra-atrial (OR: $1.12,95 \%$ CI: $1.01-1.24 ; p=0.041$ ), and interatrial EMD (OR: $1.08,95 \%$ CI: $1.01-1.16 ; p=0.026)$ were found to be independent predictors of PD (Table 3).

\section{Discussion}

The major findings of the present study are (1) patients with newly diagnosed PD are more likely to have abnormal atrial conduction times as assessed by prolonged PWD, PAs, PAl, PAt, intra-atrial, and interatrial EMD; (2) prolonged atrial conduction times were significantly correlated with the severity of PD as assessed by the UPDRS score, and (3) PWD, left intra-atrial, and interatrial EMD were found to be independent predictors of PD. 
TABle 1: Baseline demographic and clinical data of the study patients.

\begin{tabular}{|c|c|c|c|}
\hline Characteristics & $\mathrm{PD}(n=51)$ & Control $(n=31)$ & $p$ value \\
\hline Age, years & $68.1 \pm 10.4$ & $67.2 \pm 13.5$ & 0.819 \\
\hline Male, $n(\%)$ & $36(71)$ & $16(52)$ & 0.135 \\
\hline Hyperlipidemia, $n$ (\%) & $12(24)$ & $8(26)$ & 0.816 \\
\hline $\mathrm{DM}, n(\%)$ & $8(16)$ & $5(16)$ & 0.958 \\
\hline Hypertension, $n(\%)$ & $14(27)$ & $11(35)$ & 0.604 \\
\hline Smoking, $n(\%)$ & $9(18)$ & $4(13)$ & 0.796 \\
\hline BMI $\left(\mathrm{kg} / \mathrm{m}^{2}\right)$ & $23.5 \pm 2.7$ & $23.0 \pm 2.5$ & 0.356 \\
\hline $\operatorname{BSA}\left(\mathrm{m}^{2}\right)$ & $1.94 \pm 0.1$ & $1.93 \pm 0.12$ & 0.659 \\
\hline \multicolumn{4}{|l|}{ Medications } \\
\hline ASA, $n(\%)$ & $6(12)$ & $5(16)$ & 0.820 \\
\hline ACEI, $n(\%)$ & $5(10)$ & $5(16)$ & 0.617 \\
\hline $\mathrm{ARB}, n(\%)$ & $5(10)$ & $7(23)$ & 0.206 \\
\hline Calcium channel blocker, $n(\%)$ & $7(14)$ & $5(16)$ & 0.765 \\
\hline Diuretic, $n(\%)$ & $7(14)$ & $8(25)$ & 0.281 \\
\hline Statin, $n(\%)$ & $8(16)$ & $6(19)$ & 0.900 \\
\hline $\mathrm{OAD}, n(\%)$ & $5(10)$ & $5(16)$ & 0.617 \\
\hline Insulin, $n(\%)$ & $4(8)$ & $3(10)$ & 0.773 \\
\hline $\mathrm{SBP}(\mathrm{mmHg})$ & $132.7 \pm 19.8$ & $135.5 \pm 14.1$ & 0.498 \\
\hline DBP (mmHg) & $78.9 \pm 8.4$ & $83.1 \pm 12.1$ & 0.065 \\
\hline HR (bpm) & $80 \pm 11$ & $79 \pm 14$ & 0.750 \\
\hline PWD (ms) & $44.9 \pm 6.1$ & $40.0 \pm 5.5$ & $<0.001$ \\
\hline
\end{tabular}

PD, Parkinson's disease; DM, diabetes mellitus; BMI, body mass index; BSA, body surface area; ASA, acetylsalicylic acid; ACEI, angiotensin converting enzyme inhibitor; ARB, angiotensin receptor blocker; OAD, oral antidiabetic; SBP, systolic blood pressure; DBP, diastolic blood pressure; HR, heart rate; PWD, p-wave dispersion.

TABLE 2: Echocardiographic data and atrial conduction time intervals of the study patients.

\begin{tabular}{lccc}
\hline Characteristics & $\begin{array}{c}\text { PD } \\
(n=51)\end{array}$ & $\begin{array}{c}\text { Control } \\
(n=31)\end{array}$ & $\begin{array}{c}p \\
\text { value }\end{array}$ \\
\hline LVDD (mm) & $44.6 \pm 4.2$ & $45.6 \pm 2.9$ & 0.232 \\
LVSD (mm) & $26.5 \pm 4.9$ & $27.2 \pm 3.3$ & 0.481 \\
IVS (mm) & $9.9 \pm 1.9$ & $9.3 \pm 1.6$ & 0.137 \\
PW (mm) & $9.1 \pm 1.3$ & $8.8 \pm 0.8$ & 0.179 \\
LV EF (\%) & $60(6)$ & $60(5)$ & 0.782 \\
LA volume index $\left(\mathrm{cm}^{3} / \mathrm{m}^{2}\right)$ & $17.1 \pm 6.4$ & $13.6 \pm 2.5$ & 0.006 \\
DT (ms) & $277(91)$ & $239(67)$ & 0.058 \\
IRT (ms) & $98.5 \pm 15.9$ & $102.6 \pm 17.7$ & 0.279 \\
E/A ratio & $0.9 \pm 0.4$ & $1.1 \pm 0.4$ & 0.057 \\
\hline Atrial conduction times & & & \\
ML-PA (ms) & $63.1 \pm 15.0$ & $49.5 \pm 9.0$ & $<0.001$ \\
MS-PA (ms) & $47.3 \pm 9.9$ & $35.0 \pm 7.3$ & $<0.001$ \\
TL-PA (ms) & $55(25)$ & $37(10)$ & $<0.001$ \\
Intra-LA EMD (ms) & $19(10)$ & $14(8)$ & $<0.001$ \\
Intra-RA EMD (ms) & $15(15)$ & $5(7)$ & $<0.001$ \\
Interatrial EMD (ms) & $18(19)$ & $13(12)$ & 0.002 \\
\hline
\end{tabular}

LVDD, left ventricular diastolic diameter; LVSD, left ventricular systolic diameter; IVS, interventricular septum thickness; PW, posterior wall thickness; $\mathrm{LV}$, left ventricle; EF, ejection fraction; LA, left atrium; A, mitral inflow late diastolic velocity; E, mitral inflow early diastolic velocity; DT, left ventricular deceleration time; IRT, isovolumic relaxation time; ML-PA, mitral lateral annulus PA duration; MS-PA; mitral septal annulus PA duration; TL-PA, tricuspid lateral annulus PA duration; EMD, electromechanical delay; RA, right atrium.

Atrial fibrillation is the most common sustained arrhythmia in clinical practice [14]. Electrophysiological studies have revealed prolonged atrial conduction times as predictors of atrial fibrillation $[15,16]$. In a previous study examining atrial conduction times with TDE, Deniz et al. have found significant correlations regarding left intra-atrial and interatrial conduction times detected by TDE and by electrophysiological study [9]. PWD and left intra-atrial conduction time detected by TDE were found to be independent predictors of inducibility of sustained atrial fibrillation in their study. Sequential analysis of atrial electromechanical coupling to evaluate the mechanisms of paroxysmal atrial fibrillation showed that atrial electromechanical coupling at the interventricular septum, left lateral mitral annulus, and right lateral tricuspid annulus was significantly longer in patients with paroxysmal atrial fibrillation (with or without underlying heart disease) compared to control subjects [7]. Left intraatrial EMD was significantly prolonged even after correction for age in patients with atrial fibrillation [7]. The juxtaposition of atrial fibrotic lesions with normal atrial fibers has been suggested as a mechanism for nonhomogeneity of atrial conduction in atrial fibrillation [17]. Prolongation of atrial electromechanical coupling might be due to the time delay from atrial electric activation to myocardial contraction and/or left atrial enlargement [8]. In our study, PAs, PAl, and PAt durations were significantly prolonged and interatrial, right intra-atrial, and left intra-atrial EMD were significantly longer in patients with newly diagnosed PD. LA volume index was also increased in patients with PD.

Previous clinical studies have shown electrocardiographic PWD to be a predictor of paroxysmal atrial fibrillation [10]. PWD reflects inhomogeneous atrial conduction via variation in $\mathrm{p}$-wave duration between different surface ECG leads $[18,19]$. PWD was significantly prolonged in PD patients in our study. Furthermore, we demonstrated that PWD had significant correlations with left intra-atrial and interatrial EMD durations in PD patients. 


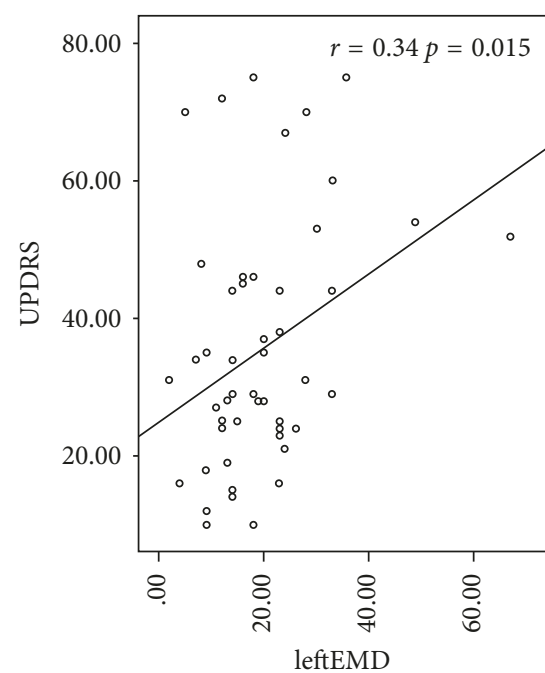

(a)

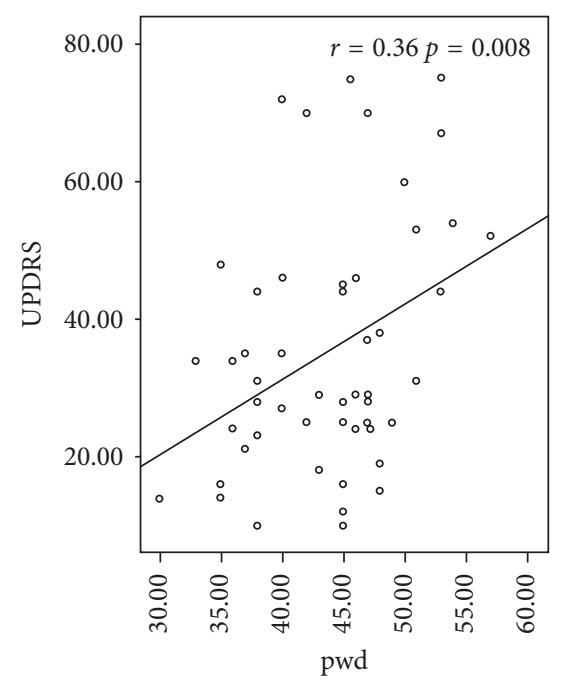

(b)

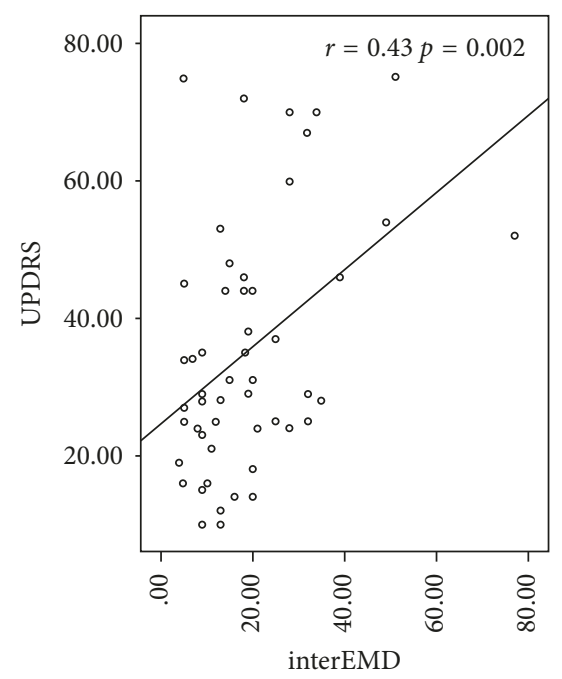

(c)

FIgURE 1: (a) Correlation between left intra-atrial EMD and the severity of Parkinson's disease (UPDRS score). (b) Correlation between p-wave dispersion and the severity of Parkinson's disease. (c) Correlation between interatrial EMD and the severity of Parkinson's disease UPDRS, Unified Parkinson's Disease Rating Scale; EMD, electromechanical delay.

TABLE 3: Multivariate analyses of variables associated with Parkinson's disease.

\begin{tabular}{lccc}
\hline Variables & OR & $95 \%$ CI & $p$ value \\
\hline PWD & 1.13 & $1.02-1.25$ & 0.017 \\
LA volume index & 1.19 & $1.02-1.37$ & 0.021 \\
Intra-LA EMD & 1.12 & $1.01-1.24$ & 0.041 \\
Interatrial EMD & 1.08 & $1.01-1.16$ & 0.026 \\
Intra-RA EMD & 1.15 & $1.05-1.25$ & 0.001 \\
\hline
\end{tabular}

OR, odds ratio; CI, confidence interval; PWD, p-wave dispersion; LA, left atrium; EMD, electromechanical delay; RA, right atrium.

Patients with atrial fibrillation have an increased risk of stroke even after adjustment for risk factors [5]. PD has also been related with an increased risk of stroke [1-3]. Therefore, prediction of development of atrial fibrillation might be vital for risk stratification of PD patients with regard to ischemic stroke. Indeed, prolonged atrial conduction times were found to be significantly correlated with the severity of PD in our study.

Several potential explanations may be postulated for the pathophysiologic mechanisms by which atrial fibrillation and PD may be related. First, both atrial fibrillation and PD are associated with an increased inflammatory state [20-23]. Neuroinflammation plays an important role in the pathogenesis of PD and contributes to the progressive loss of nigral dopaminergic neurons [20]. Inflammatory responses mediated by activated glial cells, $\mathrm{T}$ cell infiltration, and increased expression of inflammatory cytokines are described as features of PD [20]. Likewise, the prevalence and prognosis of atrial fibrillation have been associated with increased levels of inflammatory markers [21, 22]. Moreover, high-sensitivity C-reactive protein levels have shown significant positive correlations with stroke risk factors in atrial fibrillation patients and have been associated with a composite endpoint of ischemic stroke, myocardial infarction, and death [23]. Second, oxidative stress plays an important role in the pathogenesis of atrial fibrillation and PD [24, 25]. Oxidative stress has been shown to increase dopamine cell degeneration in PD [24]. Increased oxidative stress measured by the redox potentials of glutathione has been found to be an independent predictor of atrial fibrillation after adjustment for risk factors, heart failure, coronary artery disease, and high-sensitivity C-reactive protein levels [25]. Third, dysfunction of the autonomic nervous system is a common occurrence in atrial fibrillation and PD [26-29]. Symptoms of cardiovascular dysautonomia such as orthostatic hypotension have been frequently reported in PD [26]. Likewise, increased vagal tone has been related to the onset of paroxysmal atrial fibrillation both through cholinergic and noncholinergic pathways $[27,28]$. An interaction between sympathetic and parasympathetic nervous system demonstrated via recording activities from stellate ganglia and vagal nerve has been shown to play a role in the development of atrial fibrillation [29].

A limitation of our study might be that the study population was not followed up in terms of development of atrial fibrillation. Another limitation might be that data regarding inflammatory and oxidative markers were not studied. Also because of the limited number of patients included in the study, our findings require validation and further studies with larger patient groups with follow-up for arrhythmias are needed.

In conclusion, the present study showed that atrial conduction times were prolonged in patients with newly diagnosed PD. Furthermore, atrial conduction parameters were significantly correlated with the severity of PD. Our findings might contribute to risk stratification of PD patients with regard to atrial fibrillation.

\section{Data Availability}

The data used to support the findings of this study are available from the corresponding author upon request. 


\section{Disclosure}

The manuscript was presented as an oral presentation at "14th International Congress of Update in Cardiology and Cardiovascular Surgery" and published as abstract at the supplement of The American Journal of Cardiology (Volume 121, Issue 8, Supplement, 15 April 2018, Page e54).

\section{Conflicts of Interest}

The authors declare that they have no conflicts of interest.

\section{References}

[1] C. Becker, S. S. Jick, and C. R. Meier, "Risk of stroke in patients with idiopathic Parkinson's disease," Parkinsonism \& Related Disorders, vol. 16, no. 1, pp. 31-35, 2010.

[2] Y. Ben-Shlomo and M. G. Marmot, "Survival and cause of death in a cohort of patients with parkinsonism: possible clue to etiology?," Journal of Neurology, Neurosurgery \& Psychiatry, vol. 58, no. 3, pp. 293-299, 1995.

[3] J. M. Gorel, C. C. Johnson, and B. A. Rybicki, "Parkinson's disease and its comorbid disorders: an analysis of Michigan mortality data, 1970 to 1990 ," Neurology, vol. 44, no. 10, pp. 1865-1868, 1994.

[4] Y.-P. Huang, L.-S. Chen, M.-F. Yen et al., "Parkinson's disease is related to an increased risk of ischemic stroke-a populationbased propensity score-matched follow-up study," PLoS One, vol. 8, no. 9, Article ID e68314, 2013.

[5] P. A. Wolf, T. R. DAwber, H. E. Thomas Jr., and W. B. Kannel, "Epidemiologic assessment of chronic atrial fibrillation and risk of stroke: The Framingham Study," Neurology, vol. 28, no. 10 , pp. 973-977, 1978.

[6] T. A. Manolio, R. A. Kronmal, G. L. Burke, D. H. O’leary, and T. R. Price, "Short-term predictors of incident stroke in older adults: the Cardiovascular Health Study," Stroke, vol. 27, no. 9, pp. 1479-1486, 1996.

[7] Q. Q. Cui, W. Zhang, H. Wang et al., "Assessment of atrial electromechanical coupling and influential factors in nonrheumatic paroxysmal atrial fibrillation," Clinical Cardiology, vol. 31, no. 2, pp. 74-78, 2008.

[8] W. Omi, H. Nagai, M. Takamura et al., "Doppler tissue analysis of atrial electromechanical coupling in paroxysmal atrial fibrillation," Journal of the American Society of Echocardiography, vol. 18, no. 1, pp. 39-44, 2005.

[9] A. Deniz, D. Y. Sahin, and M. Kanadasi, "Conduction characteristics in atrial fibrillation," Herz, vol. 39, no. 1, pp. 137-141, 2014.

[10] S. Okutucu, K. Aytemir, and A. Oto, "P-wave dispersion: what we know till now?," JRSM Cardiovascular Disease, vol. 5, article 2048004016639443, 2016.

[11] A. J. Hughes, S. E. Daniel, L. Kilford, and A. J. Lees, "Accuracy of clinical diagnosis of idiopathic Parkinson's disease: a clinico-pathological study of 100 cases," Journal of $\mathrm{Neu}$ rology, Neurosurgery and Psychiatry, vol. 55, no. 3, pp. 181184, 1992.

[12] Movement Disorder Society Task Force on Rating Scales for Parkinson's Disease, “The Unified Parkinson's Disease Rating Scale (UPDRS): status and recommendations," Movement Disorders, vol. 18, no. 7, article 738750, 2003.

[13] R. M. Lang, M. Bierig, F. A. Flachskampf et al., "Recommendations for chamber quantification: a report from the American society of Echocardiography's guidelines and standards committee and the chamber quantification writing group, developed in conjunction with the European association of Echocardiography, a branch of the European Society of Cardiology," Journal of the American Society of Echocardiography, vol. 18, no. 12, pp. 1440-1463, 2005.

[14] C. R. C. Wyndham, "Atrial fibrillation: the most common arrhythmia," Texas Heart Institute Journal, vol. 27, no. 3, pp. 257-267, 2000.

[15] JC. Daubert, D. Pavin, G. Jauvert, and P. Mabo, "Intra- and interatrial conduction delay: implications for cardiac pacing," Pacing and Clinical Electrophysiology, vol. 27, no. 4, pp. 507-525, 2004.

[16] P. Papageorgiou, K. Monahan, N. G. Boyle et al., "Sitedependent intra-atrial conduction delay: relationship to initiation of atrial fibrillation," Circulation, vol. 94, no. 3, pp. 384-389, 1996.

[17] M. Allessie, J. Ausma, and U. Schotten, "Electrical, contractile and structural remodeling during atrial fibrillation," Cardiovascular Research, vol. 54, no. 2, pp. 230-246, 2002.

[18] P. E. Dilaveris and J. E. Gialafos, "P-wave dispersion: a novel predictor of paroxysmal atrial fibrillation," Annals of Noninvasive Electrocardiology, vol. 6, no. 2, pp. 159-165, 2001.

[19] P. E. Dilaveris, E. J. Gialafos, S. K. Sideris et al., "Simple electrocardiographic markers for the prediction of paroxysmal idiopathic atrial fibrillation," American Heart Journal, vol. 135, no. 5, pp. 733-738, 1998.

[20] K. U. Tufekci, R. Meuwissen, S. Genc, and K. Genc, "Inflammation in Parkinson's disease," Advances in Protein Chemistry and Structural Biology, vol. 88, pp. 69-132, 2012.

[21] Y. Guo, G. Y. Lip, and S. Apostolakis, "Inflammation in atrial fibrillation," Journal of the American College of Cardiology, vol. 60, no. 22, pp. 2263-2270, 2012.

[22] J. A. Vilchez, V. Roldan, D. Hernandez-Romero, M. Valdes, G. Y. Lip, and F. Marin, "Biomarkers in atrial fibrillation: an overview," International Journal of Clinical Practice, vol. 68, no. 4, pp. 434-443, 2014.

[23] J. Hermida, F. L. Lopez, R. Montes, K. Matsushita, B. C. Astor, and A. Alonso, "Usefulness of high-sensitivity C-reactive protein to predict mortality in patients with atrial fibrillation," American Journal of Cardiology, vol. 109, no. 1, pp. 95-99, 2012.

[24] P. Jenner, "Oxidative stress in Parkinson's disease," Annals of Neurology, vol. 53, no. S3, pp. S26-S38, 2003.

[25] A. Samman Tahhan, P. B. Sandesara, S. S. Hayek et al., "Association between oxidative stress and atrial fibrillation," Heart Rhythm, vol. 14, no. 12, pp. 1849-1855, 2017.

[26] T. Ziemssen and H. Reichmann, "Cardiovascular dysautonomia in de novo Parkinson's disease," Journal of the Neurological Sciences, vol. 289, no. 1-2, pp. 74-80, 2010.

[27] H. E. Hoff and L. A. Geddes, "Cholinergic factor in auricular fibrillation," Journal of Applied Physiology, vol. 8, no. 2, pp. 177-192, 1955.

[28] D. Yang, Y. Xi, T. Ai et al., "Vagal stimulation promotes atrial electrical remodeling induced by rapid atrial pacing in dogs: evidence of a noncholinergic effect," Pacing and Clinical Electrophysiology, vol. 34, no. 9, pp. 1092-1099, 2011.

[29] P. S. Chen, L. S. Chen, M. C. Fishbein et al., "Role of the autonomic nervous system in atrial fibrillation: pathophysiology and therapy," Circulation Research, vol. 114, no. 9, pp. 1500-1515, 2014. 


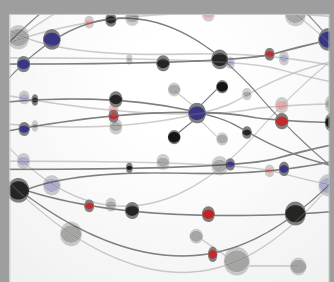

The Scientific World Journal
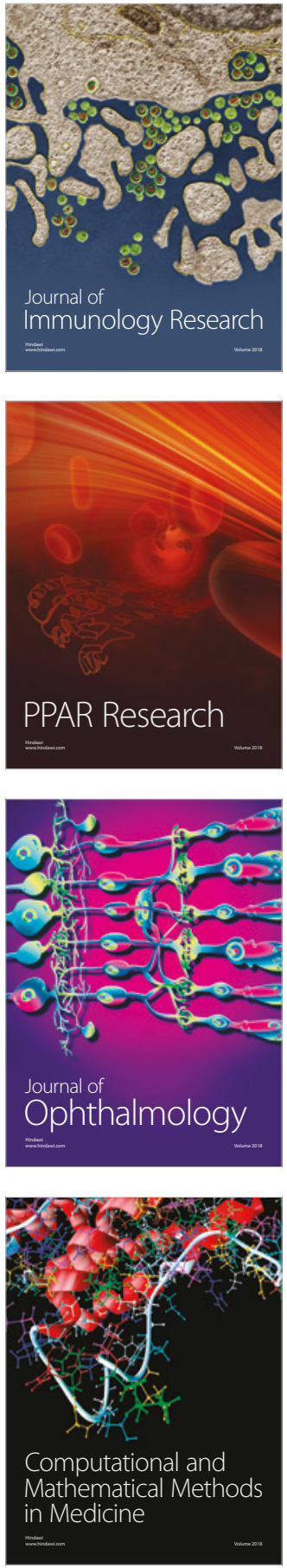

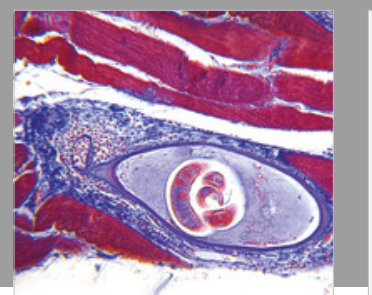

Gastroenterology Research and Practice

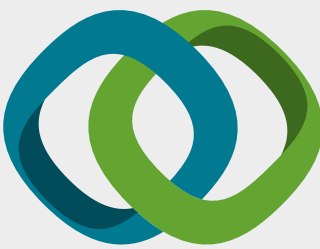

\section{Hindawi}

Submit your manuscripts at

www.hindawi.com
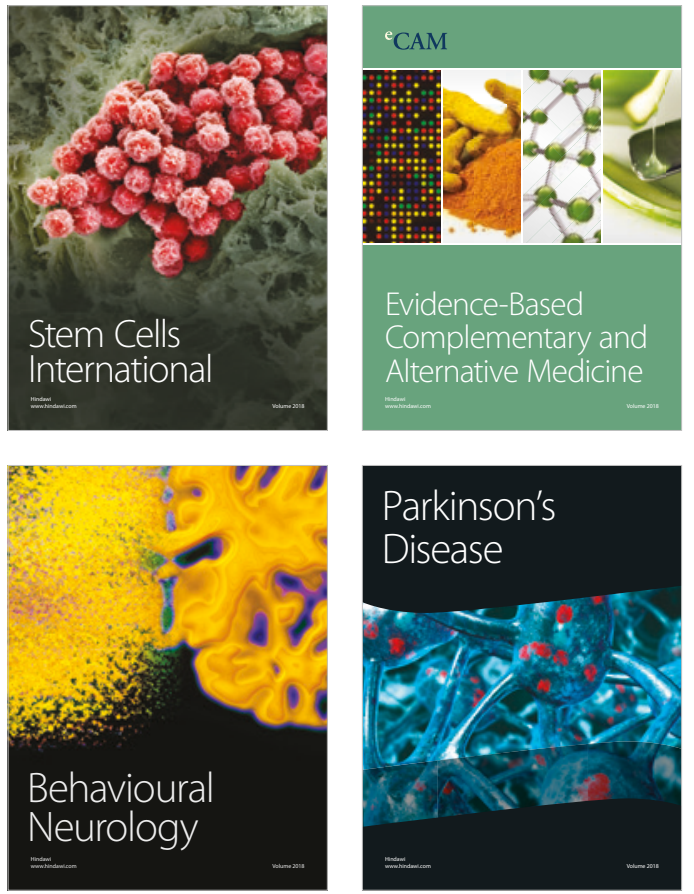

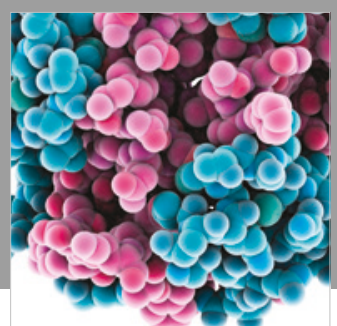

ournal of

Diabetes Research

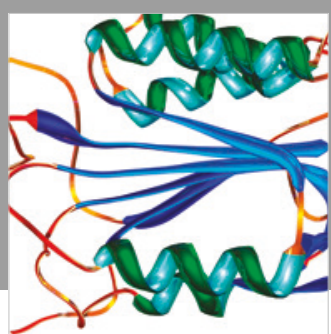

Disease Markers
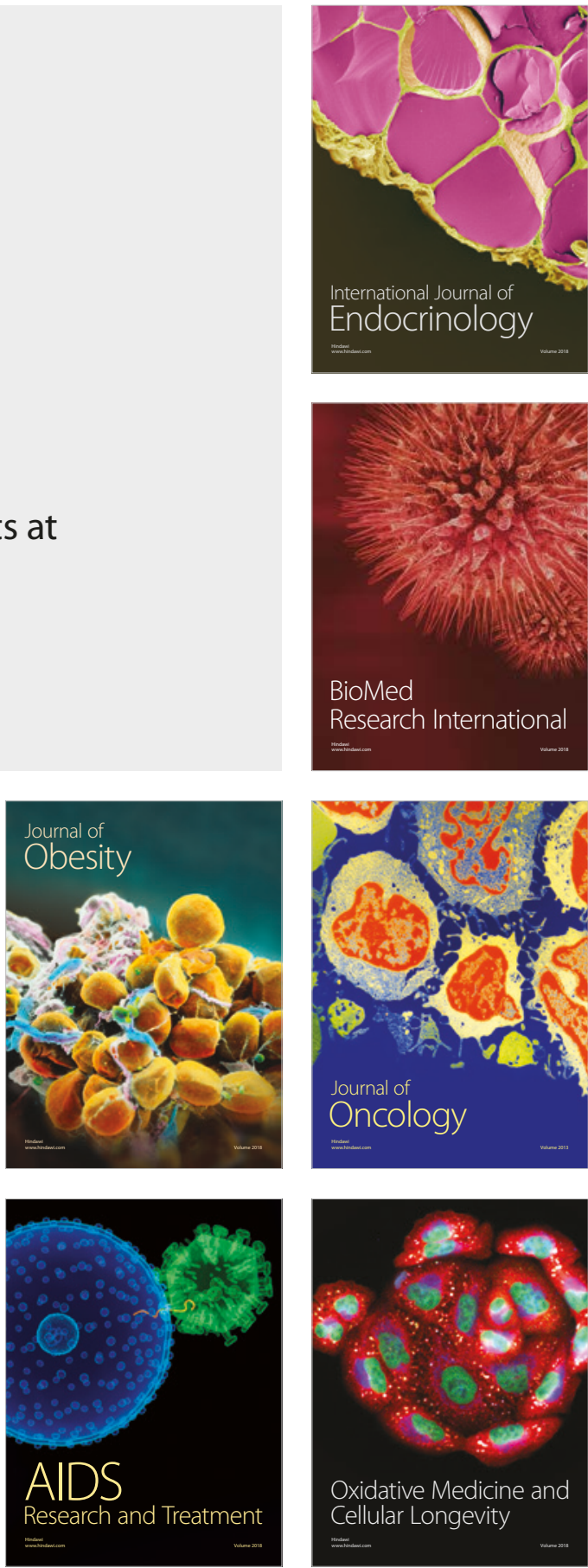